\title{
ARTICLE
}

\section{Ultrafast and reversible control of the exchange interaction in Mott insulators}

\author{
J.H. Mentink ${ }^{1, \dagger}$, K. Balzer ${ }^{1} \&$ M. Eckstein ${ }^{1}$
}

The strongest interaction between microscopic spins in magnetic materials is the exchange interaction $J_{\text {ex }}$. Therefore, ultrafast control of $J_{\mathrm{ex}}$ holds the promise to control spins on ultimately fast timescales. We demonstrate that time-periodic modulation of the electronic structure by electric fields can be used to reversibly control $J_{\text {ex }}$ on ultrafast timescales in extended antiferromagnetic Mott insulators. In the regime of weak driving strength, we find that $J_{\text {ex }}$ can be enhanced and reduced for frequencies below and above the Mott gap, respectively. Moreover, for strong driving strength, even the sign of $J_{\text {ex }}$ can be reversed and we show that this causes time reversal of the associated quantum spin dynamics. These results suggest wide applications, not only to control magnetism in condensed matter systems, for example, via the excitation of spin resonances, but also to assess fundamental questions concerning the reversibility of the quantum many-body dynamics in cold atom systems.

\footnotetext{
${ }^{1}$ Max Planck Research Department for Structural Dynamics, University of Hamburg-CFEL, 22761 Hamburg, Germany. $\dagger$ Present address: Radboud University, Institute for Molecules and Materials, Heyendaalseweg 135, 6525 AJ Nijmegen, The Netherlands. Correspondence and requests for materials should be addressed to J.H.M. (email: j.mentink@science.ru.nl).
} 
C ontrolling magnetically ordered systems on subpicosecond timescales is currently a widely studied research area owing to the joint fundamental interest and technological demand for faster and more energy-efficient magnetic storage ${ }^{1}$. The fastest pathways to reverse magnetic order utilize the exchange interaction $J_{\mathrm{ex}}$ between microscopic magnetic moments ${ }^{2-6}$, which can exceed external magnetic fields by orders of magnitude. Because $J_{\text {ex }}$ relies on the electrostatic Coulomb repulsion and the Pauli principle rather than on magnetic dipole forces, it may be modified directly by the action of a laser pulse on the electronic state. This implies an appealing and largely unexplored scenario to control magnetism on the fastest possible timescale. Recently, several experimental studies on magnetic materials have discussed an ultrafast modification of $J_{\mathrm{ex}}$ or change of the type of magnetic order by creating a nonequilibrium electron distribution (by photodoping or laser heating $)^{7-15}$. In these cases the spin dynamics after the excitation strongly depends on the relaxation of the electrons, thereby hindering a direct and reversible control of the spin degrees of freedom alone. On the other hand, reversible electrical control of $J_{\text {ex }}$ was recently demonstrated in a multiferroic solid state system, where the bond alignment can be changed by a static electric field ${ }^{16}$. Clearly, a natural goal is to achieve control of $J_{\mathrm{ex}}$, which is both reversible and ultrafast, that is, it is active while a laser pulse is on, but leaves the electronic state unexcited after the pulse is switched off.

A versatile approach to reversibly control the dynamics of quantum systems is given by the rectification of time-periodic perturbations. The use of periodic driving to control the dynamics of a quantum system is known in many areas of physics, for example, through effective conservative forces resulting from the AC Stark effect or through the coherent destruction of tunnelling ${ }^{17,18}$. For extended solid state systems, it is well known that particles in a tight-binding band subject to periodic driving evolve under an effective Hamiltonian that has a different band structure ${ }^{17-22}$. On the other hand, the control of the exchange interaction requires to understand how the driving influences both the band structure and the electronic correlations, which determine $J_{\mathrm{ex}}$ in equilibrium. This is a highly challenging problem in general, since it implies the solution of a strongly time-dependent many-body problem of an extended system.

In recent years, important insights into the control of $J_{\mathrm{ex}}$ have been obtained by studying the effect of periodic driving for one and two spin systems, leading to light-induced Kondo effects ${ }^{23}$, the design of an effective low-energy spin Hamiltonian in bosonic double-well systems ${ }^{24}$, as well as an optically induced RKKY interaction between localized spins in semiconductor quantum dots by virtual excitation of delocalized excitons ${ }^{25,26}$. The latter has been shown to be effective in extended systems as well, in particular for the dilute ferromagnetic (FM) semiconductors ${ }^{27,28}$. However, since the spin dynamics in ferromagnets requires a change of the total angular momentum, it is difficult to induce fast dynamics by modifying $J_{\mathrm{ex}}$. To the contrary, extended antiferromagnetic (AFM) systems do not suffer from the angular momentum bottleneck, and hence can provide novel opportunities for the ultrafast control of the spin dynamics by modifying $J_{\mathrm{ex}}$.

In this paper, we demonstrate that it is possible to reversibly control $J_{\mathrm{ex}}$ in extended AFM Mott insulators by periodically modulating the electronic structure with a frequency $\omega$ higher than $J_{\mathrm{ex}} / \hbar$, but not resonant to electronic excitations. We investigate a simple driving scheme using time-periodic electric fields, which can be realized both in solid state systems and for cold atoms, and hence suggests wide applications: besides the possibility of manipulating magnetism in solids, for example, via the excitation of spin resonances, we find that in the extreme limit of strong driving one may even achieve a sign reversal of $J_{\text {ex }}$, which is equivalent of letting the system evolve backwards in time and may allow for addressing fundamental questions concerning the reversibility of quantum many-body dynamics ${ }^{29-31}$ in cold atom experiments. Furthermore, we show that considerable insight can be obtained from analytical Floquet theory for a few-site cluster under continuous driving, which predicts reversible enhancement, reduction and even complete signchange of the exchange interaction. The relevance of these results for extended many-body systems may not be clear a priori, since in this case a true quasi-steady driven state may always become infinitely excited ${ }^{32}$. For the relatively short-term dynamics of interest here, the predictions of Floquet theory are nevertheless correct, as we demonstrate using numerical calculations for both the classical spin dynamics in highdimensional Mott insulators and the quantum spin dynamics in low-dimensional Mott insulators.

\section{Results}

Floquet theory for a two-site cluster. In this work, we study the repulsive Hubbard model as a model for strongly interacting electrons on a lattice. The Hamiltonian is given by

$$
H=-t_{0} \sum_{\langle i j\rangle \sigma} c_{i \sigma}^{\dagger} c_{j \sigma}+U \sum_{j} n_{j \uparrow} n_{j \downarrow},
$$

where $c_{i \sigma}^{\dagger}$ creates an electron at site $i$ with spin $\sigma=\uparrow, \downarrow, t_{0}$ is the hopping between nearest-neighbour sites, and $U$ the repulsive on-site interaction. Arbitrary time-dependent electric fields $\mathbf{E}(t)$ are incorporated by adding a Peierls phase to the hopping matrix elements (see Methods). Below we set $\hbar=1$ and measure energy and time in units of the hopping $t_{0}$ and the inverse hopping, respectively. Electric fields are measured in units of $t_{0} / e a$, where $a$ is the lattice spacing and $e$ the electron charge.

For half-filling and $U / t_{0} \gg 1$, the Hubbard model describes a Mott insulator with one electron per site, in which the remaining spin degrees of freedom are coupled by an AFM exchange interaction $J_{\mathrm{ex}}=2 t_{0}^{2} / U$. The simplest analytical understanding for this result is obtained already for two electrons on two Hubbard sites: for total $S_{z}=0$ we have four states. In the atomic limit, two of them $(|\uparrow, \downarrow\rangle$ and $|\downarrow, \uparrow\rangle)$ are singly occupied sites at $E_{1}=0$, while the other two states involve a doubly occupied and empty site at energy $E_{2}=U(|\uparrow \downarrow, 0\rangle$ and $|0, \uparrow \downarrow\rangle)$. In the presence of hopping, the degeneracy is lifted and the lowest states become singlet and triplet states at energies $E_{S}=-4 t_{0}^{2} / U$ and $E_{T}=0$, respectively. The low-energy spectrum is thus described by a spin Hamiltonian $2 J_{\mathrm{ex}} \mathbf{S}_{1} \mathbf{S}_{2}$ with $J_{\mathrm{ex}}=\left(E_{T}-E_{S}\right) / 2=2 t_{0}^{2} / U$. This analytical understanding from the cluster is useful since lattice effects beyond the lowest-order perturbative result only appear in the order $t_{0}^{4} / U^{3}$. In the same spirit, to gain theoretical insight into the modification of $J_{\mathrm{ex}}$ by periodic driving, we first consider the same two-site Hubbard cluster and employ Floquet's theorem $^{33,34}$, the analogue of Bloch's theorem in time. When the Hamiltonian is periodic in time with a period $T=2 \pi / \omega$, solutions of the time-dependent Schrödinger equation are given in the form $|\psi(t)\rangle=e^{-\mathrm{i} \epsilon_{\alpha} t}\left|\psi_{\alpha}(t)\right\rangle$, where $\left|\psi_{\alpha}(t+T)\right\rangle=\left|\psi_{\alpha}(t)\right\rangle$ is time periodic and $\epsilon_{\alpha}$ is a quasi-energy defined up to multiples of $\omega$. The Floquet picture describes a system that undergoes virtual absorption and emission of an arbitrary number of photons, as depicted in Fig. 1c for the Mott-Hubbard systems. The unperturbed Floquet sectors are described by the time-averaged Hamiltonian shifted by $n \omega$, and mixing between these Floquet sectors results in a renormalization of quasi-energy levels. A natural procedure is then to adopt an 'adiabatic' principle in which the driving amplitude varies slowly as compared with the driving frequency, $|\dot{E} / E| \ll \omega$, and identify the 

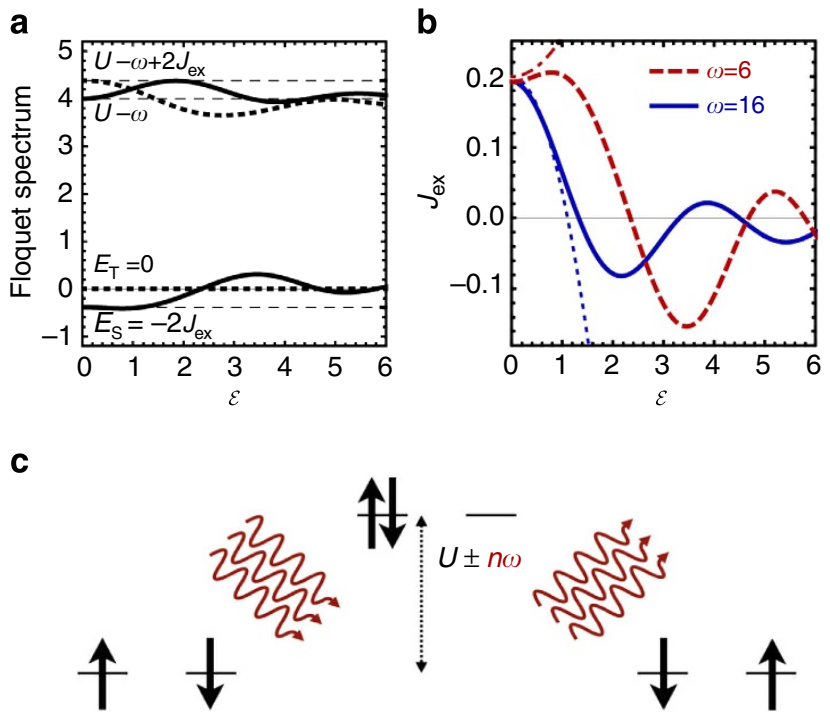

Figure 1 | Floquet spectrum of a two-site Hubbard model. (a) Floquet spectrum for $U=10$ and $\omega=6$ as a function of Floquet amplitude $\mathcal{E}$. Thin dashed lines indicate the spectrum without driving. The exchange interaction $\left(J_{\text {ex }}\right)$ is extracted from the difference between the lowest singlet $\left(E_{S}\right)$ and triplet $\left(E_{T}\right)$ levels. The result is shown in $\mathbf{b}$ for the same $U=10$ and two different frequencies above ( $\omega=16$, blue solid line) and below ( $\omega=6$, red dashed line) the Mott gap. For large driving strength $\mathcal{E} \gtrsim 1$, a reversal of $J_{\text {ex }}$ is possible. In addition, thin blue dotted $(\omega=16)$ and thin red dash dotted $(\omega=6)$ lines indicate the modification of $J_{\text {ex }}$ as obtained within second-order perturbation theory. (c) Illustration of the modification of $J_{\text {ex }}$ in the Floquet picture. Red wavy lines indicate the virtual absorption and emission of an arbitrary number $n$ of photons with frequency $\omega$. This induces a coupling to excited states in different Floquet sectors displaced by an energy $U \pm n \omega$.

amplitude-dependent singlet-triplet splitting $\epsilon_{T}-\epsilon_{S}$ in the quasienergy spectrum with the (time dependent) exchange interaction that describes the spin dynamics in the laser driven system on timescales much slower than the driving period $T$.

The numerical solution of the Floquet spectrum and various analytically tractable limiting cases for the two-site Hubbard model are detailed in the methods section. For a tight-binding model driven by an electric field $E(t)=E_{0} \cos (\omega t)$, the coupling between Floquet sectors is controlled by the dimensionless Floquet amplitude

$$
\mathcal{E}=\frac{e a E_{0}}{\hbar \omega},
$$

and the time averaging of $H$ corresponds to a coherent reduction of the tunnelling amplitude ${ }^{17,18}$ by a factor $J_{0}(\mathcal{E})$, where $J_{0}$ is the Bessel function (see Methods). In Fig. 1, we display the Floquet spectrum and the exchange splitting $J_{\text {ex }}(\mathcal{E})$ for a half-filled twosite Hubbard model. In contrast to the limit $\omega \gg U, t_{0}$, where the only effect would be a renormalization of the hopping by $J_{0}(\mathcal{E})$ and a corresponding reduction of the exchange splitting at large $U$ by a factor $J_{0}(\mathcal{E})^{2}$, one can see that $J_{\text {ex }}$ can be both increased and decreased for finite $\omega$, depending on the driving. This is clear already in the perturbative limit for $\mathcal{E} \ll 1$ and $t_{0} / U \ll 1$, which is given by $J_{\mathrm{ex}}=2 t_{0}^{2} / U+\Delta J_{\mathrm{ex}}$ with (see Methods)

$$
\Delta J_{\mathrm{ex}}=\frac{\mathcal{E}^{2} t_{0}^{2}}{4}\left(\frac{1}{U+\omega}+\frac{1}{U-\omega}-\frac{2}{U}\right),
$$

and indicated with thin (dash-)dotted lines in the right panel of Fig. 1. The last term of equation (3) is the reduction of the exchange due to coherent reduction of the tunnelling, while the first two terms derive from the coupling to the $m= \pm 1$ Floquet sector with effectively shifted charge-transfer energies $U \pm \omega$. The net effect is an enhancement (reduction) of $J_{\mathrm{ex}}$ for driving frequencies below (above) the Coulomb energy $U$. For sufficiently strong driving one can even reverse the sign of $J_{\mathrm{ex}}$, thus leading to the remarkable finding of a FM exchange coupling in the halffilled Hubbard model. This happens when $\mathcal{E}$ is of order one, such that coupling to higher Floquet sectors with effective Coulomb energy $U-m \omega$ becomes strongly enhanced, while the direct exchange path is reduced by coherent destruction of tunnelling $\sim J_{0}(\mathcal{E})$. For larger Floquet amplitudes, the direct exchange path again increases due to the oscillating behaviour of the Bessel function. In the remainder of this paper, we verify that these predictions from the two-site Floquet picture can remain valid for extended condensed matter systems at off-resonant driving with finite pulse duration, in spite of the possible importance of higher-order processes such as multi-photon absorption in the strongly driven regime, and the limited number of cycles in the pulse.

Mean-field spin dynamics in a high-dimensional lattice. A direct prediction of the Floquet theory (equation (3)) is the enhancement (reduction) of the exchange interaction for driving below (above) the Mott gap with weak amplitudes $(\mathcal{E} \ll 1)$. A large class of materials for which this might be relevant are threedimensional bulk systems, such as transition metal oxides, whose low-energy spin dynamics contain uniform spin resonances that can be conveniently described in mean-field theory. For large dimensions, also a numerical solution of the nonequilibrium electron dynamics in the Hubbard model is possible using the dynamical mean-field theory (DMFT, see Methods).

Within DMFT, the equilibrium solution of the Hubbard model at half-filling and low temperature is the Néel state. To asses the exchange interaction in this state, we study the excitation of resonances in the AFM phase in a transverse magnetic field $B_{x}$, a setup that was pioneered in ref. 15 for resonant photoexcitation. In equilibrium, the balance of $B_{x}$ and $J_{\text {ex }}$ gives rise to a canting of the magnetic sublattices out of the $y-z$ plane. If $J_{\mathrm{ex}}$ is modified under periodic driving, the sub-lattice magnetizations are no longer aligned with the effective field $B_{\text {eff }}$ given by external and exchange fields, as illustrated in the inset of Fig. 2. This implies a rotation of the spins in the plane perpendicular to $B_{x}$ (leaving the total angular momentum $S_{x}$ conserved), from which the timedependent modification of the exchange interaction is calculated (see Methods). Hence, in our calculations, $J_{\mathrm{ex}}$ is defined as the parameter that describes best the observed transverse spin dynamics as is obtained from the solution of the full electron problem. We stress that this allows us to quantify changes of the exchange interaction independent of the exchange energy stored in the system. This is particularly important for the regime in which absorption is not negligible, where the laser excites mobile carriers that transfer their energy to the spin background on an ultrafast timescale and thus reduce the ordered moment.

We have implemented the DMFT solution of the Hubbard model in a time-dependent external electric field for the infinitedimensional hyper-cubic lattice with density of states $D(\epsilon)=$ $\exp \left(-\epsilon^{2}\right) / \sqrt{\pi}$ (refs 35,36). The electric field is pointing along the body diagonal of the lattice and represents a laser pulse with frequency $\omega$ and a Gaussian envelope that contains 15 cycles per pulse, that is, $E(t)=E_{0} \sin (\omega t) \exp \left(-\left(t-3 t_{c}\right)^{2} / t_{c}^{2}\right)$ with $t_{c}=15 \pi /(2.1 \omega)$. Figure $2 \mathrm{a}, \mathrm{b}$ show time traces of the induced change of the exchange interaction $\Delta J_{\mathrm{ex}}^{c}(t)$ for one driving frequency below (a) and above (b) gap, as extracted from the time evolution of the spin degrees of freedom during the pulse. In accordance with the prediction of the Floquet theory, we observe an enhancement (reduction) of the exchange interaction during 

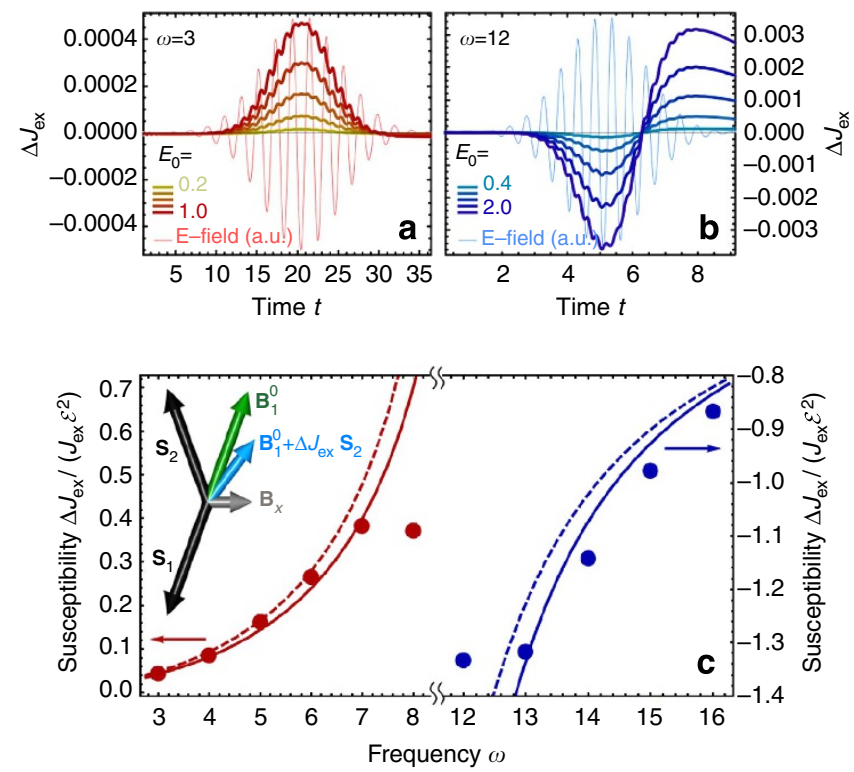

Figure 2 | Laser-induced modification of the exchange interaction. (a,b) Time-dependent change of the exchange interaction ( $\Delta J_{\text {ex }}$, thick lines) during the action of a laser pulse, for driving frequencies $\omega=3$ (a) and $\omega=12$ (b) below and above the Mott gap, respectively. Different colours correspond to results obtained with different amplitude $E_{0}$ of the electric field, increasing from light to dark. Thin lines show the time dependence of the electric field (E-field). Numerical results were obtained using dynamical mean-field theory (DMFT) for the hyper-cubic lattice at $U=10$ and initial temperature $T=0.025$. (c) The driving susceptibility $\Delta J_{\mathrm{ex}} /\left(J_{\mathrm{ex}} \mathcal{E}^{2}\right)$ for $\mathcal{E} \rightarrow 0$ for frequencies above (blue, right vertical axis) and below gap (red, left vertical axis), obtained from DMFT for the hyper-cubic lattice (disks), from the numerical Floquet spectrum of a two-site Hubbard cluster (solid lines), and from the perturbative result equation (3) (dashed lines). The inset illustrates the canted geometry of the two sub-lattice magnetizations $\mathbf{S}_{1,2}$ (black arrows) induced by a static transverse magnetic field $\mathbf{B}_{\mathrm{x}}$ (grey arrow). In equilibrium, the effective magnetic field $\mathbf{B}_{1}^{0}$ (green arrow) is collinear with $\mathbf{S}_{1}$. A modification of the exchange interaction $\left(\Delta J_{\mathrm{ex}}\right)$ would rotate the effective field (light blue arrow) with respect to $\mathbf{S}_{1}$ causing the excitation of a spin resonance. In the DMFT calculations, $\Delta J_{\text {ex }}$ is computed from the observed spin precession in this canted geometry.

the application of the field with a frequency below (above) gap. The frequency $\omega=3$ in Fig. $2 \mathrm{a}$ is far from the resonance $\omega \approx U$, and we observe that $\Delta J_{\mathrm{ex}}^{c} \approx 0$ after the pulse, demonstrating the reversibility of the effect. Conversely, the driving frequency $\omega=12$ in Fig. $2 \mathrm{~b}$ is chosen close to the edge of the upper Hubbard band where we observe significant absorption and transient behaviour after the pulse. Hence, although the exchange interaction is modified in this case as well, the effect is not reversible. Note also that the time reached in the present simulations is too short for the photoexcited carriers to relax, hence a description in terms of a quasi-stationary photo-doped state discussed earlier ${ }^{15}$ is not yet valid.

A quantitative comparison with the Floquet theory is shown in the bottom panel of Fig. 2, where the 'driving susceptibility' $\Delta J_{\mathrm{ex}} /\left(J_{\mathrm{ex}} \mathcal{E}^{2}\right)$ for $\mathcal{E} \rightarrow 0$ is plotted as a function of the driving frequency. Solid discs show the DMFT results as obtained by a linear fit through the dependence of the ratio $\Delta J_{\mathrm{ex}}(t) / J_{\mathrm{ex}}$ on $\mathcal{E}^{2}$ at its maximum. Dashed and solid lines show the results based on the perturbative Floquet formula (equation (3)) and the full Floquet spectrum (non-perturbative in $t_{0} / U$ ), evaluated from the derivative $d J_{\mathrm{ex}} / d \mathcal{E}^{2}$ at $\mathcal{E}=0$. As expected, in the vicinity of the band edge $(|\omega-U| \sim 2)$, strong deviation is found since band absorption is not captured in a cluster picture. Away from the band edge, however, the frequency dependence matches very well, being even in quantitative agreement for the lowest frequencies below gap. This demonstrates the usefulness of the Floquet theory for understanding how off-resonant periodic driving modifies the exchange interaction in extended condensed matter systems by photo-assisted hopping.

One-dimensional quantum spin dynamics. An intriguing prediction of the Floquet analysis is the existence of amplitude and frequency ranges in which the exchange coupling becomes FM. Such a sign-change of $J_{\mathrm{ex}}$ cannot cause a transition to a FM state since the Hubbard model equation (1) conserves the total spin. However, even if the system remains AFM, a change of sign of $J_{\mathrm{ex}}$ by periodic driving allows for a very non-trivial and unique way to control the spin dynamics, namely, to reverse the time evolution of the undriven system. Such time reversal can be anticipated by considering a pure Heisenberg spin Hamiltonian $H_{\mathrm{ex}}=J_{\mathrm{ex}} \sum_{\langle i j\rangle} \mathbf{S}_{i} \mathbf{S}_{j}$, which gives an accurate description of the low-energy spin dynamics in the half-filled Hubbard model at $U \gg t_{0}$ if the system is not electronically excited. In the absence of driving, the propagation over a time interval $t$ is given by the evolution operator $\mathcal{U}_{\mathrm{AFM}}=\exp \left(-\mathrm{i} H_{\mathrm{ex}} t\right)$. Such evolution can exactly be reversed by the propagation with an exchange interaction $J_{\mathrm{ex}}^{\prime}$ of opposite sign over a time interval $t^{\prime}=\left|J_{\mathrm{ex}} / J_{\mathrm{ex}}^{\prime}\right| t$, since for the FM time evolution operator we have $\mathcal{U}_{\mathrm{FM}}=\exp \left(-\mathrm{i} H_{\mathrm{ex}}^{\prime} t^{\prime}\right)=\exp \left(+\mathrm{i} H_{\mathrm{ex}} t\right)=\mathcal{U}_{\mathrm{AFM}}^{-1}$, that is, the two time evolution operators are exactly inverse to each other.

To demonstrate that periodic driving of the Hubbard model at large $U$ indeed yields the anticipated time reversal of the spin degrees of freedom, we consider a chain of $L=10$ sites and compute the dynamics using exact diagonalization techniques (see Methods). The system is initially prepared in a classical Néel state $c_{1 \uparrow}^{\dagger} c_{2 \downarrow}^{\dagger} c_{3 \uparrow}^{\dagger} \ldots|0\rangle$ and is evolved under the unperturbed Hamiltonian (1). In a quantum Heisenberg model, the classical Néel state is a highly excited state the energy of which exceeds the thermal energy at the Néel temperature, such that no remanent long-range order is expected at long times, apart from finite size effects. In one dimension, not even the ground state displays long-range order. As a consequence of the spin-flip terms $J_{\mathrm{ex}}\left(S_{i}^{+} S_{i+1}^{-}+S_{i}^{-} S_{i+1}^{+}\right)$in the effective AFM Heisenberg model, we thus observe a rapid decay of the total staggered magnetization $M=\frac{1}{L} \sum_{i=1}^{L}(-1)^{i+1}\left\langle n_{i \uparrow}-n_{i \downarrow}\right\rangle$ (Fig. 3b). After this initial free evolution to a state where long-range order is suppressed, we ramp on a time-periodic electric field (Fig. 3a), with Floquet amplitude $\mathcal{E}=3.4$ and frequency $\omega / U=0.6$ such that the Floquet theory for a two-site model predicts a reversal of the exchange coupling. Under the periodic driving, one indeed observes a near perfect reversal of the dynamics of $M(t)$ in Fig. 3b, which almost completely recovers to the initial value $M(t=0)$ around $t \approx 100$. Subsequently, $M(t)$ is reduced again by further evolution in the reverse direction, as a consequence of the spin-flip terms in the FM model. This continues until the field is ramped off, after which one observes that the free evolution brings the system again back to the initial state, from which the same rapid decay of $M(t)$ is observed as for the initial free evolution. Hence, we conclude that the periodic driving allows for a reversible control of the spin dynamics for the timescale considered in our simulations. This is further confirmed by the time evolution of the total double occupation $d=\sum_{i=1}^{L}\left\langle n_{i \uparrow} n_{i \downarrow}\right\rangle$, which has the same mean value before and after the driving, demonstrating that electronic excitations due to the driving are negligible. The weak oscillations in $d(t)$ are caused by switching on the hopping at 


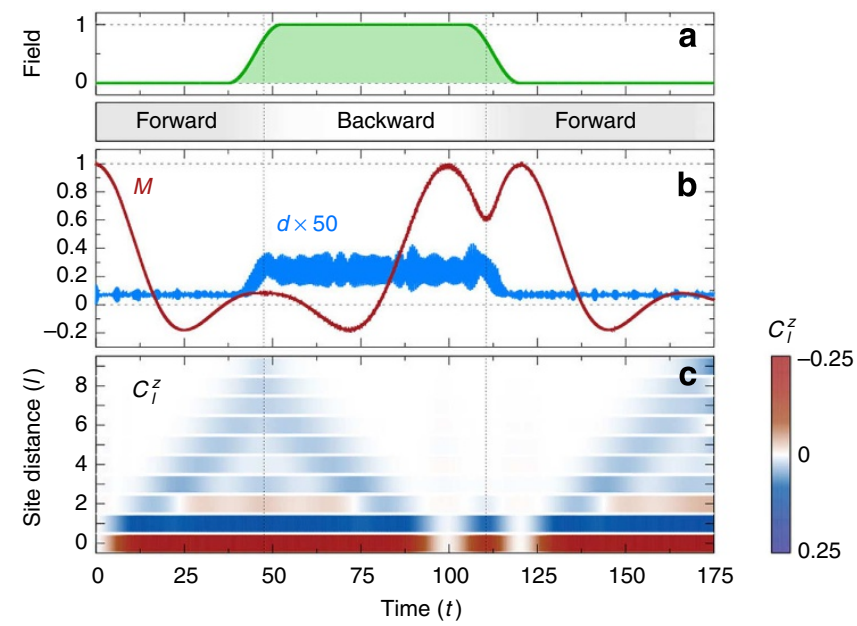

Figure 3 | Time reversal of the spin dynamics by periodic driving. (a) Field envelope with cosine-shaped ramps of length $\Delta t=15$ around $t_{\mathrm{f}}=45$ and $t=112.5$. The bar below the field envelope indicates forward (grey) and backward (white) time evolution when the field is off and on, respectively. (b) Time evolution of staggered magnetization $M$ (red) and total double occupation $d$ (blue), scaled by a factor of 50 , of a 10 -site Hubbard chain, showing free evolution for times $t<37.5$ and $t>120$ and evolution under an additional periodic driving at frequency $\omega / U=0.6$ and Floquet amplitude $\mathcal{E}=3.4$ in between. (c) Build up and diminishing of the spin-spin correlation function $C_{l}^{z}$. Numerical results were obtained by exact diagonalization for $U=50$ and open boundary conditions, starting from a classical Néel state. The colour bar indicates the value of $C_{l}^{2}$, ranging from -0.25 (dark red) through 0 (white) to 0.25 (dark blue).

$t=0$, while the increased mean value of $d(t)$ during driving is due to photo-assisted hopping processes.

Time reversal can be demonstrated not only on the level of local observables. Figure $3 \mathrm{c}$ displays the evolution of the spinspin correlation function $C_{l}^{z}=\sum_{|i-j|=l} \frac{1}{N_{l}}\left(\left\langle S_{i}^{z} S_{j}^{z}\right\rangle-\left\langle S_{i}^{z}\right\rangle\left\langle S_{j}^{z}\right\rangle\right)$ as a function of distance $l$ and time $\left(N_{l}\right.$ is the number of site pairs with distance $l$ ). Starting from the initial uncorrelated product state, correlations build up under the evolution of $H$. Even though the system is small, the spreading of correlations resembles the lightcone effect that has been observed in quantum many-body systems after a quench ${ }^{37}$, that is, correlations stay zero outside the light cone $|l| \leq 2 v t$, where $v$ is a maximal mode velocity ${ }^{38}$, while short-range AFM correlations emerge inside the light cone. Further, under the action of the periodic driving, the spin-spin correlations diminish with the same speed, restoring the initially uncorrelated state.

To determine quantitatively how well the time evolution is reversed in our simulations, we computed the difference $\Delta M=1$ $-M\left(t^{\star}\right)$ between the initial magnetization in the Néel state and the magnetization $M\left(t^{\star}\right)$ at the revival time $t^{\star}$ for different values of $U$ (Fig. 4). In all simulations, the system is evolved forward in time for a given time $t_{\mathrm{f}}=10$, after which the field is ramped on for a period $\Delta t=10$. As before, we choose $\mathcal{E}=3.4$ and $\omega=0.6 \mathrm{U}$, which gives the same relative change of $J_{\mathrm{ex}}$ for all sufficiently large values $U \gg t_{0}$. The observed scaling $\Delta M \sim 1 / U^{2}$ indicates that in the current setup the deviation from perfect reversal originates from small electronic excitations above the gap, which arises from switching on the hopping in the beginning of the simulation and the ramping on of the field. Since the dynamics of the electronically excited states is not captured by the spin Hamiltonian, it is not time-reversed. While the electronic excitation can be further reduced by slow ramping, the ultimate limit is given by non-Heisenbergian terms in the effective spin a

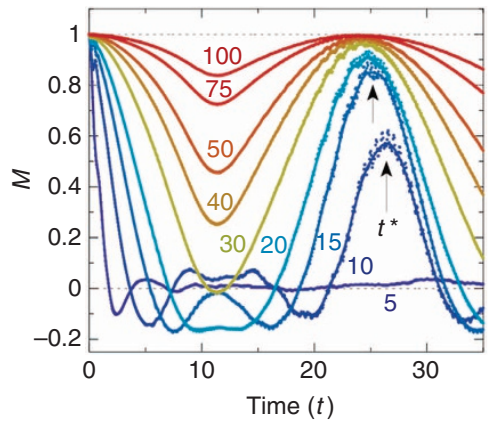

b

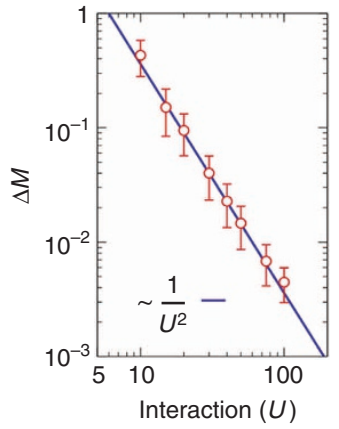

Figure 4 | Quality of the time reversal. (a) Time evolution of the staggered magnetization $M$ in a 10-site Hubbard chain, where after fixed forward propagation time $t_{\mathrm{f}}=10$ the field is ramped up within a time interval $\Delta t=10$. Different colours correspond to different values of $U$, as indicated by the corresponding coloured labels. (b) Difference $\Delta M$ between the initial staggered magnetization and the staggered magnetization at the revival time $t^{\star}$ as a function of $U$. The error bars represent the magnitude of the short-time fluctuations of $M$ close to $t^{\star}$. In all calculations, the driving frequency $\omega / U=0.6$ and Floquet amplitude $\mathcal{E}=3.4$ were used.

Hamiltonian, for which perfect reversal under periodic driving is not expected. For the half-filled Hubbard model in equilibrium, the leading-order correction to the Heisenberg model appears in the order $t_{0}^{4} / U^{3}$ in the strong-coupling expansion. At least for small times $t$, the contribution of a perturbation $\delta H$ proportional to $t_{0}^{4} / U^{3}$ to the time-reversed Hamiltonian would lead to a scaling $\Delta M \sim 1 / U^{3}$, which is smaller than the electronic excitation in the present case. Note, however, that the times reached in the current simulations are nevertheless long enough to observe near perfect reversal even from a state without magnetic order, in which spin correlations have spread throughout the full chain (Fig. 3).

\section{Discussion}

Our results demonstrate ultrafast and reversible electrical control of the exchange interaction in extended fermionic many-body systems by modulation with time-periodic electric fields. These numerical results have recently been corroborated analytically in the high-frequency limit, by showing that the effective spin model for the two-site cluster remains valid for the driven lattice ${ }^{39}$. We emphasize that Floquet amplitudes $\mathcal{E} \sim 0.1$ are well accessible for condensed matter systems, which would lead to relative changes of $J_{\mathrm{ex}}$ up to $1 \%$. For example, for a frequency $\hbar \omega=1 \mathrm{eV}$ and a lattice spacing of $2 \AA$, a laser fluence of $1 \mathrm{~mJ} \mathrm{~cm}^{-2}$ in a $100-\mathrm{fs}$ pulse corresponds to a Floquet parameter $\mathcal{E} \sim 0.05$. While realistic condensed matter systems usually involve several correlated bands, we think that our current results are already quite robust for the AFM oxides governed by superexchange interactions. Similar as in the single-band model studied here, superexchange interactions are governed by virtual charge excitations, which will be reversibly modified through the mechanism of photo-assisted hopping between different Floquet sectors. This is further supported by recently presented experiments on canted AFM oxides ${ }^{40}$ using terahertz emission spectroscopy ${ }^{41}$, which show the first experimental evidence of reversibly controlling exchange interactions by off-resonant pumping below the charge-transfer gap. Furthermore, equation (3) implies a strong enhancement of off-resonant effects at low frequencies. As an extreme limit of this one can anticipate control of spin dynamics by few-cycle terahertz pulses, using both the coupling between the spins to the magnetic field of the light ${ }^{42}$, and the modifications of $J_{\text {ex }}$ predicted by our work. At the same time, extensions to multi-band models are very 
interesting and relevant to perform as they usually involve multiple possibly competing exchange paths, which generally yield quantitative differences as well as different dependencies on the driving frequency due to the presence of additional resonances, potentially enabling to achieve even stronger effects. In this connection, we also mention that extensions to multi-band models may be relevant too for the description of metallic ferromagnets. Here static electronic structure calculations already indicate that exchange integrals involving electrons excited to higher bands considerably differ from those in the ground state ${ }^{43}$. Furthermore, a more accurate quantitative description of photoexcited states would also include the effect of dynamic screening. However, we anticipate that this will be important only if mobile carriers are injected, that is, for photo-doping excitations, while it will be a secondary effect for the off-resonant driving investigated here, which leaves the electron distribution unchanged.

While the single-band model is clearly a minimal model for application to condensed matter systems, fermionic cold atom systems resemble the single-band model very accurately. Moreover, such systems may realize the large amplitudes $\mathcal{E}$ needed to achieve the FM exchange at frequencies sufficiently far from resonances $n \omega=U$, which are difficult to realize for most condensed matter systems. It will, therefore, be of fundamental interest to investigate the reversal of the exchange interaction and the associated time reversal of the quantum spin dynamics in cold atom experiments. In these systems recently, great progress has been made to prepare and measure systems with single-site spatial resolution ${ }^{44,45}$. Furthermore, various single-particle Floquet Hamiltonians could be realized in the limit of strong periodic driving without substantial heating by inter-band absorption (see, for example, ref. 46). Cold atoms in optical lattices have been successfully used as a quantum simulator for the dynamics of a quantum quenches in the Bose-Hubbard model, starting from an artificially prepared charge-ordered phase ${ }^{47}$, which suggest similar techniques to probe the behaviour of spin systems under time reversal. In the methods section (cf. equation (7)), we show that analogous time reversal can be achieved by modulating the amplitude of the hopping instead of its phase, which is easier to control in cold atoms. An intriguing problem to study both theoretically and experimentally is the fundamental question how well the time evolution can be reversed after (much) longer forward evolution time and investigate systematically the influence of small deviations from perfect time reversal. Furthermore, a study of the Loschmidt echo and dynamical phase transitions ${ }^{48}$ in cold atoms might be possible by including additional perturbations to the back propagation.

\section{Methods}

Floquet theory. The Floquet quasi-energy spectrum can be obtained from the ansatz $|\psi(t)\rangle=e^{-\mathrm{i} \epsilon_{\alpha} t}\left|\psi_{\alpha}(t)\right\rangle$ by expanding $\left|\psi_{\alpha}(t)\right\rangle$ in a Fourier series

$\left|\psi_{\alpha}(t)\right\rangle=\sum_{m} e^{i \omega m t}\left|\psi_{\alpha, m}\right\rangle$, where $\left|\psi_{\alpha, m}\right\rangle$ is referred to as the component of the wave function in the $m$ th Floquet sector. The Schrödinger equation then achieves a block-matrix structure

$$
\left(\epsilon_{\alpha}+m \omega\right)\left|\psi_{\alpha, m}\right\rangle=\sum_{m^{\prime}} H_{m-m^{\prime}}\left|\psi_{\alpha, m^{\prime}}\right\rangle
$$

where $H_{m}=(1 / T) \int_{0}^{T} d t e^{\mathrm{i} \omega m} H(t)$ are the Fourier components of the Hamiltonian. Different from the usual discussions of Floquet theory for single-particle Hamiltonians, here we focus on the effect of periodic driving on an electronic spectrum that is controlled by electronic correlations.

Time-dependent electric fields are incorporated into the Hubbard Hamiltonian (1) by adding a time-dependent Peierls phase to the hopping matrix elements, $t_{i j}(t)=t_{0} \exp \left[\mathrm{i} e a A_{i j}(t)\right]$, where $A_{i j}$ is the projection of the vector potential along the direction from site $i$ to $j$ (choosing a gauge with zero scalar potential and $\left.E(t)=-\partial_{t} A(t)\right)$. For the one-dimensional chain with electric field $E_{0} \sin (\omega t)$ along the chain, this implies $A_{i j}(t)=-\frac{1}{\omega}(i-j) E_{0} \cos (\omega t)$. The Fourier components of the Hamiltonian are thus given by

$$
H_{m}=-t_{0} \sum_{\langle i j\rangle \sigma}(-1)^{m} J_{m}((i-j) \mathcal{E}) c_{i \sigma}^{\dagger} c_{j \sigma},
$$

plus the additional (time-independent) interaction part in the $m=0$ component, where $J_{m}(x)$ is the $m$ th Bessel function, and the dimensionless parameter $\mathcal{E}=e a E_{0} /(\hbar \omega)$ measures the strength of the perturbation (cf. equation (2)). For the numerical determination of the Floquet energies, one truncates the number of Floquet sectors in equation (4) to $|n| \leq N$, and increases $N$ to reach convergence. The determination of a many-body Floquet spectrum thus requires the diagonalization of a matrix of dimension $N \times D$, where $D$ is the dimension of the Hilbert space. The results presented in Fig. 1 are converged with $N=8$.

In the limit of large frequency, $\omega \gg U, t_{0}$, Floquet sectors in equation (4) are separated in energy, and one can restrict oneself to the lowest sector $m=0$. This is equivalent to replacing the Hamiltonian with its time average, which leads to the renormalization of the hopping by $J_{0}(\mathcal{E})$, and a corresponding reduction of the exchange by a factor $J_{0}(\mathcal{E})^{2}$. In the perturbative limit where both $t_{0} / U \ll 1$ and $\mathcal{E} \ll 1$, we expand the Bessel functions $J_{n}(x) \sim x^{n}$ for $x \rightarrow 0$. To lowest order only states of the $m=0$ and $m= \pm 1$ Floquet sectors have to be taken into account, and the result given by equation (3) follows from standard second-order perturbation theory. Furthermore, an interesting limit for the Mott regime is given by $t_{0} / U \ll 1$, but allowing for fields of arbitrary amplitude. Because all terms $H_{m}$ for $m \neq 0$ are proportional to $t_{0}$, the perturbative shift of the spin states in the $m=0$ sector is given by a sum over all second-order processes containing precisely one virtual hopping to a higher Floquet sector and back, yielding

$$
\frac{J_{\mathrm{ex}}(\mathcal{E}, \omega)}{J_{\mathrm{ex}}(\mathcal{E}=0)}=\sum_{n=-\infty}^{\infty} \frac{J_{|n|}(\mathcal{E})^{2}}{1+n \omega / U} .
$$

The unperturbed exchange is modified by a factor dependent only on $\omega / U$ and $\mathcal{E}$. For the parameters $\mathcal{E}=3.4$ and $\omega / U=0.6$ chosen for the time reversal, for example, the factor is given by -0.95 and indicates a near perfect sign reversal.

Finally, we note that a similar analysis is possible for the case of periodic modulation of the hopping amplitude, taking $t_{0}(t)=t_{0}(1+A \cos (\omega t))$. As above, we obtain for $U \ll t$

$$
\frac{J_{\mathrm{ex}}(A, \omega)}{J_{\mathrm{ex}}(A=0)}=1+\sum_{n= \pm 1} \frac{A^{2}}{1+n \omega / U},
$$

yielding, for example, a perfect sign reversal for driving above gap $\omega / U=1.2$ at $A \approx 0.67$. Other than for the field driven case, the perturbation is purely harmonic, and only Floquet sectors $m= \pm 1$ enter this expression.

Dynamical mean-field theory. To solve the electron dynamics in the Hubbard model, we use nonequilibriumDMFT ${ }^{35,49}$. The electric field of the laser is incorporated by the Peierls substitution (see Methods, Floquet theory), so that the light matter interaction within the single-band model is treated to all orders. Within DMFT ${ }^{50}$, which becomes exact in the limit of infinite dimensions ${ }^{51}$, local correlation functions are obtained from an effective impurity model in which one site of the lattice is coupled to a non-interacting, self-consistently determined bath. The impurity model is solved within the perturbative hybridization expansion (non-crossing approximation ${ }^{52}$ ). The accuracy of this approach has been tested in equilibrium and for the short-time dynamics by comparison with higher-order hybridization expansions, as well as with numerically exact Quantum Monte Carlo ${ }^{52-54}$, which revealed good agreement both in the AFM and paramagnetic Mott insulator regime $t_{0} \ll U$. A detailed description of the formalism and of our numerical implementation is given in ref. 15, which studies the same setup (that is, a hyper-cubic lattice with electric field along the body diagonal), yet for different electric fields.

Determination of $\boldsymbol{J}_{\mathbf{e x}}$ in DMFT. To asses the exchange interaction from mean field spin dynamics, we investigate the AFM phase of the Hubbard model supplemented with a term $B_{x} \sum_{i} S_{i x}$, which couples the spin $S_{i \alpha}=\frac{1}{2} \sum_{\sigma \sigma^{\prime}} c_{i \sigma}^{\dagger}\left(\hat{\sigma}_{\alpha}\right)_{\sigma \sigma^{\prime}} c_{i \sigma^{\prime}}$ to a homogeneous magnetic field $B_{x}$ along the $x$ axis $\left(\hat{\sigma}_{\alpha}\right.$ denotes the Pauli matrices; $\alpha=x, y, z)$. DMFT allows us to compute the time-dependent expectation value of the electron spin $\left\langle\mathbf{S}_{1,2}\right\rangle$ on the two magnetic sublattices. Assuming a rigid macrospin model, the time-dependent exchange interaction can be inferred from these results by inverting the Landau-Lifshitz equation for the dynamics of spins on the mean field $\mathbf{B}_{\text {eff }}^{1,2}=B_{x} \mathbf{e}_{x}-2 J_{\mathrm{ex}}\left\langle\mathbf{S}_{2,1}\right\rangle$. It was shown ${ }^{15}$ that this approach compares well with the definition of exchange interactions from a time-dependent response formalism ${ }^{55}$, as well as to the analytical perturbative result $J_{\mathrm{ex}}=2 t_{0}^{2} / U$ in equilibrium at large $U$. In the transverse field, the equilibrium exchange interaction can be determined from the canting induced by $B_{x}$, yielding $J_{\mathrm{ex}}^{c}=-B_{x} /\left(4\left\langle S_{x}\right\rangle\right)$. Out of equilibrium, we obtain $J_{\mathrm{ex}}^{c}(t)=-B_{x} /\left(4\left\langle S_{x}\right\rangle\right)+\Delta J_{\mathrm{ex}}^{c}(t)$,

$$
\Delta J_{\mathrm{ex}}^{c}(t)=-\frac{1}{4 T\left\langle S_{1 x}\right\rangle} \int_{t-T / 2}^{t+T / 2} \frac{\left\langle\dot{S}_{1 y}(s)\right\rangle}{\left\langle S_{1 z}(s)\right\rangle} d s,
$$

where the time averaging is done to extract only the low-frequency component, similar as in the Floquet theory. Note that by calculating the exchange interaction in this way, we have automatically projected out dynamical changes in the (time 
averaged) local magnetization $\left|\left\langle\mathbf{S}_{1,2}\right\rangle\right|$, and slight changes of the local moments (double occupation) as a result of the virtual absorption and emission of photons.

Exact diagonalization. To compute the time evolution of the (driven) one-dimensional Hubbard model from the Schrödinger equation with a time-dependent Hamiltonian $H(t)$ and a given initial state $\left|\psi_{0}\right\rangle=c_{1 \uparrow}^{\dagger} c_{2 \downarrow}^{\dagger} c_{3 \uparrow}^{\dagger} \ldots|0\rangle$, we use the Krylov technique ${ }^{56}$ in combination with a commutator-free exponential time-propagation scheme ${ }^{57}$. While the Krylov method provides efficient approximations to the time propagator, which are important to treat large Hilbert spaces, the commutator-free exponential time-propagation scheme is related to the Magnus expansion, and, preserving unitarity, allows for a high-order accurate integration of the Schrödinger equation in time.

\section{References}

1. Kirilyuk, A., Kimel, A. V. \& Rasing, Th. Ultrafast optical manipulation of magnetic order. Rev. Mod. Phys. 82, 2731-2784 (2010).

2. Radu, I. et al. Transient ferromagnetic-like state mediating ultrafast reversal of antiferromagnetically coupledspins. Nature 472, 205-208 (2011).

3. Mentink, J. H. et al. Ultrafast spin dynamics in multisublattice magnets. Phys. Rev. Lett. 108, 057202 (2012)

4. Wienholdt, S., Hinzke, D., Carva, K., Oppeneer, P. M. \& Nowak, U. Orbitalresolved spin model for thermal mag-netization switching in rare-earth-based ferrimagnets. Phys. Rev. B 88, 020406 (2013).

5. Baryakhtar, V. G., Butrim, V. I. \& Ivanov, B. A. Exchange relaxation as the mechanism of ultrafast spin re-orientation in two-sublattice ferrimagnets. JETP Lett. 98, 289-293 (2013).

6. Evans, R. F. L., Ostler, T. A., Chantrell, R. W., Radu, I. \& Rasing, Th. Ultrafast thermally induced magnetic switching in synthetic ferrimagnets. Appl. Phys. Lett. 104, 082410 (2014).

7. Rhie, H.-S., Dürr, H.A. \& Eberhardt, W. Femtosecond electron and spin dynamics in ni/w(110) films. Phys. Rev. Lett. 90, 247201 (2003).

8. Ju, G. et al. Ultrafast generation of ferromagnetic or-der via a laser-induced phase transformation in ferh thin films. Phys. Rev. Lett. 93, 197403 (2004).

9. Thiele, J., Buess, M. \& Back, C. H. Spin dynamics of the antiferromagnetic-toferromagnetic phase transition in ferh on a sub-picosecond time scale. Appl. Phys. Lett. 85, 2857 (2004).

10. Wall, S., Prabhakaran, D., Boothroyd, A. T. \& Cavalleri, A. Ultrafast coupling between light, coherent lattice vibrations, and the magnetic structure of semicovalent LaMnO3. Phys. Rev. Lett. 103, 097402 (2009).

11. Först, M. et al. Driving magnetic order in a manganite by ultrafast lattice excitation. Phys. Rev. B 84, 241104 (2011).

12. Carley, R. et al. Femtosecond laser excitation drives ferromagnetic gadolinium out of magnetic equilibrium. Phys. Rev. Lett. 109, 057401 (2012).

13. Li, T. et al. Femtosecond switching of magnetism via strongly correlated spin-charge quantum excitations. Nature 496, 69-73 (2013).

14. Matsubara, M. et al. Tuning the ultrafast spin dynamics in carrier-densitycontrolled ferromagnets. Preprint at http://arxiv.org/abs/1304.2509 (2013).

15. Mentink, J. H. \& Eckstein, M. Ultrafast quenching of the exchange interaction in a Mott insulator. Phys. Rev. Lett. 113, 057201 (2014).

16. Ryan, P. J. et al. Reversible control of magnetic inter-actions by electric field in a single-phase material. Nat. Commun. 4, 1334 (2013).

17. Dunlap, D. H. \& Kenkre, V. M. Dynamical localization of a particle moving under inuence of an electric field. Phys. Rev. B 34, 3625-3633 (1986).

18. Grossmann, F., Dittrich, T., Jung, P. \& Hänggi, P. Coherent destruction of tunneling. Phys. Rev. Lett. 67, 516-519 (1991)

19. Lignier, H. et al. Dynamical control of matter-wave tunneling in periodic potentials. Phys. Rev. Lett. 99, 220403 (2007).

20. Oka, T. \& Aoki, H. Photovoltaic Hall effect in graphene. Phys. Rev. B 79, 081406 (2009).

21. Tsuji, N., Oka, T., Werner, P. \& Aoki, H. Dynamical band ipping in fermionic lattice systems: an ac-field-driven change of the interaction from repulsive to attractive. Phys. Rev. Lett. 106, 236401 (2011).

22. Wang, Y. H., Steinberg, H., Jarillo-Herrero, P. \& Gedik, N. Observation of Floquet-Bloch states on the surface of a topological insulator. Science 342, 453-457 (2013)

23. Shahbazyan, T., Perakis, I. \& Raikh, M. Spin correlations in nonlinear optical response: Light-induced kondo effect. Phys. Rev. Lett. 84, 5896-5899 (2000).

24. Chen, Y.-A., Nascimbeńe, S., Aidelsburger, M., Atala, M., Trotzky, S. \& Bloch, I. Controlling correlated tunnel-ing and superexchange interactions with ac-driven optical lattices. Phys. Rev. Lett. 107, 210405 (2011).

25. Piermarocchi, C., Pochung Chen, L., Sham \& Steel, D. Optical RKKY interaction between charged semicon-ductor quantum dots. Phys. Rev. Lett. 89, $167402(2002)$

26. Piermarocchi, C. \& Quinteiro, G. F. Coherent optical control of spin-spin interaction in doped semiconductors. Phys. Rev. B 70, 235210 (2004).

27. Fernández-Rossier, J., Piermarocchi, C., Chen, P., Mac-Donald, A. \& Sham, L. Coherently photoinduced ferromagnetism in diluted magnetic semiconductors. Phys. Rev. Lett. 93, 127201 (2004).
28. Wang, J., Cotoros, I., Dani, K., Liu, X., Furdyna, J. \& Chemla, D. Ultrafast enhancement of ferromagnetism via photoexcited holes in GaMnAs. Phys. Rev. Lett. 98, 217401 (2007).

29. Lebowitz, J. L. Boltzmann's entropy and time's arrow. Phys. Today 46, 32-38 (1993).

30. Polkovnikov, A. Microscopic diagonal entropy and its connection to basic thermodynamic relations. Ann. Phys. 326, 486-499 (2011).

31. Caneva, T., Silva, A., Fazio, R., Lloyd, S., Calarco, T. \& Montangero, S. Complexity of controlling quantum many-body dynamics. Phys. Rev. A $\mathbf{8 9}$, 042322 (2014).

32. D'Alessio, L. \& Rigol, M. Long-time behavior of isolated periodically driven interacting lattice systems. Phys. Rev. X 4, 041048 (2014).

33. Floquet, G. Sur les équations différentielles linéaires à coefficients périodiques. Ann. Sci. Ec. Normale Super 12, 47-88 (1883).

34. Grifoni, M. \& Hänggi, P. Driven quantum tunnelling. Phys. Rep. 304, 229-354 (1998).

35. Aoki, H., Tsuji, N., Eckstein, M., Kollar, M., Oka, T. \& Werner, P. Nonequilibrium dynamical mean-field theory and its applications. Rev. Mod. Phys. 86, 779-837 (2014).

36. Turkowski, V. \& Freericks, J. K. Nonlinear response of bloch electrons in infinite dimensions. Phys. Rev. B 71, 085104 (2005).

37. Cheneau, M. et al. Light-cone-like spreading of correlations in a quantum many-body system. Nature 481, 484-487 (2012).

38. Calabrese, P. \& Cardy, J. Time dependence of correlation functions following a quantum quench. Phys. Rev. Lett. 96, 136801 (2006).

39. Itin, A. P. \& Katsnelson, M. I. Effective Hamiltonians for fastly driven manybody lattice systems. Preprint at http://arxiv.org/abs/1401.0402v2 (2015).

40. Mikhaylovskiy, R. V. et al. Inverse magneto-refraction as a mechanism for laser modification of spin-spin exchange parameters and subsequent Terahertz emission from iron oxides. Preprint at http://arxiv.org/abs/1412.7094 (2014).

41. Mikhaylovskiy, R. V., Hendry, E., Kruglyak, V. V., Pisarev, R. V. \& Kimel, Th. Rasing, and A. V. Terahertz emission spectroscopy of laser-induced spin dynamics in $\mathrm{TmFeO} 3$ and $\mathrm{ErFeO} 3$ orthoferrites. Phys. Rev. B 90, 184405 (2014)

42. Kampfrath, T. et al. Coherent Terahertz control of antiferromagnetic spin waves. Nat. Photon 5, 31-34 (2011).

43. Zhang, G. P., Gu, M. \& Wu, X. S. Ultrafast reduction in exchange interaction by a laser pulse: alternative path to femtomagnetism. J. Phys. Condens. Matter 26, 376001 (2014).

44. Bakr, W. S. et al. Probing the superuid-to-Mott insulator transition at the single-atom level. Science 329, 547-550 (2010).

45. Endres, M. et al. Single-site- and single-atom-resolved measurement of correlation functions. Appl. Phys. B 113, 27-39 (2013).

46. Struck, J. et al. Tunable gauge potential for neutral and spinless particles in driven optical lattices. Phys. Rev. Lett. 108, 225304 (2012).

47. Trotzky, S. et al. Probing the relaxation towards equilibrium in an isolated strongly correlated one-dimensional bose gas. Nat. Phys. 8, 325-330 (2012).

48. Heyl, M., Polkovnikov, A. \& Kehrein, S. Dynamical quantum phase transitions in the transverse-field Ising model. Phys. Rev. Lett. 110, 135704 (2013).

49. Freericks, J. K., Turkowski, V. M. \& Zlatić, V. Nonequilibrium dynamical mean-field theory. Phys. Rev. Lett. 97, 266408 (2006).

50. Georges, A., Kotliar, G., Krauth, W. \& Rozenberg, M. J. Dynamical mean-field theory of strongly correlated fermion systems and the limit of infinite dimensions. Rev. Mod. Phys. 68, 13-125 (1996).

51. Metzner, W. \& Vollhardt, D. Correlated lattice fermions in $d=\infty$ dimensions. Phys. Rev. Lett. 62, 324-327 (1989).

52. Eckstein, M. \& Werner, P. Nonequilibrium dynamical mean-field calculations based on the noncrossing approximation and its generalizations. Phys. Rev. B 82, 115115 (2010).

53. Eckstein, M. \& Werner, P. Photoinduced states in a Mott insulator. Phys. Rev. Lett. 110, 126401 (2013).

54. Werner, P., Tsuji, N. \& Eckstein, M. Nonthermal symmetry-broken states in the strongly interacting hubbard model. Phys. Rev. B 86, 205101 (2012).

55. Secchi, A., Brener, S., Lichtenstein, A. I. \& Katsnelson, M. I. Non-equilibrium magnetic interactions in strongly correlated systems. Ann. Phys. 333, 221-271 (2013).

56. Hochbruck, M. \& Lubich, C. On Krylov subspace ap-proximations to the matrix exponential operator. SIAM J. Numer. Anal. 34, 1911-1925 (1997).

57. Alvermann, A. \& Fehske, H. High-order commutator-free exponential time-propagation of driven quantum sys-tems. J. Comp. Phys. 230, 5930-5956 (2011).

\section{Acknowledgements}

We thank U. Bovensiepen, M.I. Katsnelson, A.V. Kimel, A. Lichtenstein, R.V. Mikhaylovskiy, T. Oka, A. Secchi, N. Tsuji and Ph. Werner for fruitful discussions. The exact diagonalization calculations were run in part on the supercomputer HLRN-II of the North-German Supercomputing Alliance. J.H.M. acknowledges funding from the Nederlandse Organisatie voor Wetenschappelijk onderzoek (NWO) by a Rubicon Grant 


\section{Author contributions}

The Floquet theory was developed by M.E. and J.H.M. DMFT simulations were run by J.H.M., while K.B. obtained the exact diagonalization results. All authors contributed to the discussion and interpretation of the results and the writing of the manuscript.

\section{Additional information}

Competing financial interests: The authors declare no competing financial interests.

Reprints and permission information is available online at http://npg.nature.com/ reprintsandpermissions/
How to cite this article: Mentink, J. H. et al. Ultrafast and reversible control of the exchange interaction in Mott insulators. Nat. Commun. 6:6708 doi: 10.1038/ ncomms7708 (2015).

(c) (i) This work is licensed under a Creative Commons Attribution 4.0 International License. The images or other third party material in this article are included in the article's Creative Commons license, unless indicated otherwise in the credit line; if the material is not included under the Creative Commons license, users will need to obtain permission from the license holder to reproduce the material. To view a copy of this license, visit http://creativecommons.org/licenses/by/4.0/ 